\title{
FINDING HAMILTONIAN ISOCHRONOUS CENTERS BY NON-CANONICAL TRANSFORMATIONS
}

\author{
M. SABAtini
}

\begin{abstract}
Starting from a class of isochronous Hamiltonian centers, we produce a new class of Hamiltonian isochronous centers by using non-canonical transformations.
\end{abstract}

2010 Mathematics Subject Classification: 34A05.

Key words: Hamiltonian system, non-canonical transformation, isochronous.

\section{Introduction}

Let $\Omega$ be an open connected subset of the real plane. Let us consider a differential system

$$
z^{\prime}=V(z), \quad z=(x, y) \in \Omega,
$$

$V(z)=\left(V_{1}(z), V_{2}(z)\right) \in C^{\omega}\left(\Omega, \mathbb{R}^{2}\right)$. We denote by $\phi_{V}(t, z)$ the local flow defined by (1). A topological annulus $A \subset \Omega$ is said to be a period annulus of (1) if it is the set-theoretical union of concentric non-trivial cycles of (1). If the inner component of $A$ 's boundary is a single point $O$, then $O$ is said to be a center, and the largest connected punctured neighbourhood $N_{O}$ of $O$ covered with non-trivial cycles is said to be its central region. If $A$ is a period annulus, we can define on $A$ the period function $T$ by assigning to each point $z \in A$ the minimum positive period $T(z)$ of the cycle $\gamma$ passing through $z$. We say that the period function $T$ is increasing if outer cycles have larger periods. We say that a period annulus $A$ is isochronous if $T$ is constant on $A$, i.e. if every cycle in $A$ has the same period.

The study of mechanical systems isochronicity was probably initiated by Galileo and Huygens, in relation to the problem of building pendulum clocks. Later on, such a problem was faced in the more general framework of differential equations. At the end of nineteenth century, Poincaré proved his celebrated theorem about systems linearization in a

This paper was partially supported by the PRIN project Equazioni differenziali ordinarie: sistemi dinamici, metodi topologici e applicazioni. 
neighbourhood of a critical point, which had as a relevant special case that of isochronous centers. The successive investigations were mainly concerned with isochronicity in conservative systems $[\mathbf{1}, \mathbf{7}, \mathbf{1 0}, \mathbf{1 2}]$,

$$
\dot{x}=y, \quad \dot{y}=-g(x) .
$$

Such systems are special cases of Hamiltonian systems with separable variables

$$
\dot{x}=f(y), \quad \dot{y}=-g(x),
$$

which were also studied in several papers. Isochronicity in more general Hamiltonian systems was also considered $[\mathbf{1}, \mathbf{2}, \mathbf{3}, \mathbf{4}, \mathbf{5}, \mathbf{6}, \mathbf{9}]$. The underlying motivation of all such papers is the fact that a Hamiltonian system is usually related to the dynamic behaviour of some physical system.

A related investigation field is that of transformations that generate new Hamiltonian systems from known ones, or, conversely, reduce the study of new Hamiltonian systems to that of known ones. The main class of such transformations is that of canonical transformations, which take every Hamiltonian system into a Hamiltonian system. A planar transformation $\Lambda$ is canonical if and only if its Jacobian determinant is a non-zero constant. In the paper [8] the existence of canonical transformations is studied in relation to isochronicity.

Another possibility, seemingly not considered so far, is that of producing new Hamiltonian systems from known ones - in particular, new isochronous systems - by means of non-canonical transformations. We do not know any general characterization of such transformations and the related systems. In this paper we give a characterization of systems obtained from Hamiltonians systems with separable variables,

$$
\dot{u}=F(v), \quad \dot{v}=-G(u),
$$

by means of transformations with separable variables, which are canonical only if linear. In order to simplify computations, we find more convenient to work on the inverse transformation, $\Psi(x, y)=(P(x), Q(y))$, that leads to write the new system as follows

$$
\dot{x}=\frac{F(Q(y))}{P^{\prime}(x)}, \quad \dot{y}=-\frac{G(P(x))}{Q^{\prime}(y)},
$$

and impose the zero-divergence condition. A simple example of such a procedure consists in transforming the linear center

$$
\dot{u}=v, \quad \dot{v}=-v,
$$


into a non-linear system

$$
\dot{x}=\frac{Q(y)}{P^{\prime}(x)}, \quad \dot{y}=-\frac{P(x)}{Q^{\prime}(y)},
$$

and imposing the zero divergence condition. This leads to find a new class os isochronous Hamiltonian systems, reversing the usual approach: rather than looking for isochronous systems in a class of Hamiltonian ones, we look for Hamiltonian systems in a class of isochronous ones.

\section{Hamiltonian systems}

We say that the function $Z$ satisfies the condition $(\zeta)$ if there exists an open interval $I_{Z}$ containing the origin 0 such that $Z \in C^{2}\left(I_{Z}, \mathbb{R}\right)$, $Z(0)=0, Z^{\prime}(t)>0$ for all $t \in I_{Z}$.

Assume $F, G, P, Q$ to satisfy $(\zeta)$. Let us consider the Hamiltonian system (2), whose Hamiltonian function is

$$
H(u, v)=\int_{0}^{u} G(s) d s+\int_{0}^{v} F(t) d t=\Gamma(u)+\Phi(v) .
$$

Setting $\Psi(x, y)=(P(x), Q(y))$, the map $\Psi^{-1}$ takes (2) into (3). In general, (3) is not a Hamiltonian system. In the next theorem we characterize the maps $\Psi$ which produce a Hamiltonian (3).

For $\kappa \in \mathbb{R}$, let us set

$$
\begin{aligned}
& \Delta_{\kappa}(p)=\int_{0}^{p} \exp \left(-\kappa \int_{0}^{u} G(s) d s\right) d u, \\
& \Xi_{\kappa}(q)=\int_{0}^{q} \exp \left(-\kappa \int_{0}^{v} F(t) d t\right) d v .
\end{aligned}
$$

Then, one has

$$
\begin{aligned}
& \Delta_{\kappa}(P(x))=\int_{0}^{P(x)} \exp \left(-\kappa \int_{0}^{u} G(s) d s\right) d u, \\
& \Xi_{\kappa}(Q(y))=\int_{0}^{Q(y)} \exp \left(-\kappa \int_{0}^{v} F(t) d t\right) d v .
\end{aligned}
$$

Theorem 1. Let F, G, P, Q satisfy $(\zeta)$. Then the system (3) is Hamiltonian if and only if there exist $\kappa_{p}, \kappa_{q} \in \mathbb{R}, \kappa_{p}, \kappa_{q}>0$, such that

$$
P(x)=\Delta_{\kappa}^{-1}\left(\kappa_{p} x\right), \quad Q(y)=\Xi_{\kappa}^{-1}\left(\kappa_{q} y\right) .
$$

Proof: Imposing the divergence of (3) to vanish one has

$$
-\frac{F(Q(y)) P^{\prime \prime}(x)}{P^{\prime}(x)^{2}}+\frac{G(P(x)) Q^{\prime \prime}(y)}{Q^{\prime}(y)^{2}}=0 .
$$


Separating variables in a neighbourhood of a point where both $G(P(x))$ and $F(Q(y))$ do not vanish, i.e. for $x y \neq 0$, one has

$$
\frac{P^{\prime \prime}(x)}{G(P(x)) P^{\prime}(x)^{2}}=\frac{Q^{\prime \prime}(y)}{F(Q(y)) Q^{\prime}(y)^{2}}, \quad x y \neq 0 .
$$

Since the above fractions depend on different variables, the equality holds if and only if there exists $\kappa \in \mathbb{R}$ such that

$$
\kappa=\frac{P^{\prime \prime}(x)}{G(P(x)) P^{\prime}(x)^{2}}=\frac{Q^{\prime \prime}(y)}{F(Q(y)) Q^{\prime}(y)^{2}}, \quad x y \neq 0 .
$$

We work on the equation involving $P(x)$, the other one can be dealt with similarly. $P(x)$ is a solution to the differential equation

$$
P^{\prime \prime}(x)=\kappa G(P(x)) P^{\prime}(x)^{2}, \quad \kappa \in \mathbb{R} .
$$

In the trivial case $\kappa=0$, one has $P^{\prime \prime}(x)=0$, which gives $P(x)=\kappa_{1} x+\kappa_{0}$. $P(0)=0$ implies $\kappa_{0}=0$, hence $P(x)=\kappa_{1} x$, with $\kappa_{1}=P^{\prime}(x)>0$. Since $\Delta_{0}$ is the identity, choosing $\kappa_{p}=\kappa_{1}$ one has $P(x)=\kappa_{p} x=\Delta_{0}^{-1}\left(\kappa_{p} x\right)$. Similarly, $Q(y)=\kappa_{q} x=\Xi_{0}^{-1}\left(\kappa_{q} x\right)$, for some positive $\kappa_{q}$.

If $\kappa \neq 0$, since $P^{\prime}(x)>0$ one can write (6) as follows,

$$
\frac{P^{\prime \prime}(x)}{P^{\prime}(x)}=\kappa G(P(x)) P^{\prime}(x) \text {. }
$$

Then one can write

$$
\begin{array}{ll}
{\left[\log \left(P^{\prime}(x)\right)\right]^{\prime}=\kappa[\Gamma(P(x))]^{\prime},} & \\
\log \left(P^{\prime}(x)\right)=\kappa \Gamma(P(x))+\kappa_{0}, & \\
P^{\prime}(x)=\exp \left(\kappa \Gamma(P(x))+\kappa_{0}\right)=\kappa_{1} \exp (\kappa \Gamma(P(x))), & \kappa_{1}>0, \\
P^{\prime}(x) \exp (-\kappa \Gamma(P(x)))=\kappa_{1}, & \kappa_{1}>0 .
\end{array}
$$

One can re-write $(7)$ as follows

$$
\left[\Delta_{\kappa}(P(x))\right]^{\prime}=\kappa_{1}, \quad \kappa_{1}>0,
$$

so that

$$
\Delta_{\kappa}(P(x))=\kappa_{1} x+\kappa_{2}, \quad \kappa_{1}>0 .
$$

$P$ and $\Delta_{\kappa}$ both vanish at 0 , hence $\kappa_{2}=0$. The function $\Delta_{\kappa}$ is invertible, since $\Delta_{\kappa}^{\prime}>0$, that implies

$$
P(x)=\Delta_{\kappa}^{-1}\left(\kappa_{1} x\right), \quad \kappa_{1}>0 .
$$

Similarly, one proves that

$$
Q(y)=\Xi_{\kappa}^{-1}\left(\kappa_{3} y\right), \quad \kappa_{3}>0 .
$$

Then, choosing $\kappa_{p}=\kappa_{1}$ and $\kappa_{q}=\kappa_{3}$, one has the statement. 
Differently from what happens with canonical transformations, even if (3) is a Hamiltonian system, its Hamiltonian function cannot be obtained from $H(u, v)$ by substituting $u$ and $v$ with $P(x)$ and $Q(y)$. In fact, the function

$$
\bar{H}(x, y)=H(P(x), Q(y))=\Gamma(P(x))+\Phi(Q(y))
$$

is a first integral of (3), but it is not its Hamiltonian function, which is

$$
K(x, y)=-\frac{1}{\kappa P^{\prime}(x) Q^{\prime}(y)} .
$$

The dynamics of (3) and (2) are equivalent, so that if (2) has an isochronous center, then also (3) has one. This is true, in particular, if (2) has a linear center. In this case system (3) has the form (5). Such a system was considered in [9] as an example of a commuting, hence isochronous, system generated by a non-singular transformation. Applying Theorem 1, we can give the explicit expression of $P(x)$ and $Q(y)$ for which (5) is Hamiltonian.

In the next statement we consider the so-called Gauss error function

$$
\operatorname{erf}(t)=\frac{2}{\sqrt{\pi}} \int_{0}^{t} e^{-u^{2}} d u
$$

and the similarly defined

$$
\overline{\operatorname{erf}}(t)=\frac{2}{\sqrt{\pi}} \int_{0}^{t} e^{u^{2}} d u .
$$

Both functions vanish at 0 and have positive derivative all over $\mathbb{R}$, hence they are globally invertible on $\mathbb{R}$.

The celebrated Poincaré Linearization Theorem states that a non-degenerate analytic center is isochronous if and only if it can be diffeomorphically transformed into a linear center. Such a result was generalized in [11] to systems of class $C^{k}, k \geq 2$. In the next corollary we characterize the isochronous Hamiltonian systems obtainable from the linear one by means of transformations with separable variables.

Corollary 1. Assume $P$ and $Q$ to satisfy the condition $(\zeta)$. Then the system (5) is Hamiltonian if and only if one of the following cases occur:

(1) both $P(x)$ and $Q(y)$ are linear;

(2) $\exists \kappa, c_{p}, c_{q}>0: P(x)=\sqrt{\frac{2}{\kappa}} \operatorname{erf}^{-1}\left(c_{p} x\right), Q(y)=\sqrt{\frac{2}{\kappa}} \operatorname{erf}^{-1}\left(c_{q} y\right)$;

(3) $\exists \kappa<0, c_{p}, c_{q}>0: P(x)=\sqrt{\frac{2}{|\kappa|}} \overline{\operatorname{erf}}^{-1}\left(c_{p} x\right), Q(y)=\sqrt{\frac{2}{|\kappa|}} \overline{\operatorname{erf}}^{-1}\left(c_{q} y\right)$. 
Proof: One has $G(u)=u, F(v)=v$, hence $\Gamma(u)=\frac{u^{2}}{2}, \Phi(v)=\frac{v^{2}}{2}$. Moreover

$$
\Delta_{\kappa}(P)=\int_{0}^{P} e^{-\kappa \frac{u^{2}}{2}} d u, \quad \Xi_{\kappa}(Q)=\int_{0}^{Q} e^{-\kappa \frac{v^{2}}{2}} d v .
$$

Integrating by means of a linear change of variables produces the statements, in the different cases $\kappa=0, \kappa>0$, and $\kappa<0$.

\section{References}

[1] J. Chavarriga and M. Sabatini, A survey of isochronous centers, Qual. Theory Dyn. Syst. 1(1) (1999), 1-70. DOI: 10.1007/ BF02969404.

[2] A. Cima, F. Mañosas, and J. Villadelprat, Isochronicity for several classes of Hamiltonian systems, J. Differential Equations 157(2) (1999), 373-413. DOI: 10.1006/jdeq.1999.3635.

[3] W. A. Coppel and L. Gavrilov, The period function of a Hamiltonian quadratic system, Differential Integral Equations 6(6) (1993), 1357-1365.

[4] E. Freire, A. Gasull, and A. Guillamon, A characterization of isochronous centres in terms of symmetries, Rev. Mat. Iberoamericana 20(1) (2004), 205-222. DOI: 10.4171/RMI/386.

[5] E. Freire, A. Gasull, and A. Guillamon, First derivative of the period function with applications, J. Differential Equations 204(1) (2004), 139-162. DOI: 10.1016/j.jde.2004.04.005.

[6] L. Gavrilov, Isochronicity of plane polynomial Hamiltonian systems, Nonlinearity 10(2) (1997), 433-448. DOI: 10.1088/0951$7715 / 10 / 2 / 008$.

[7] J. J. Levin and S. S. Shatz, Nonlinear oscillations of fixed period, J. Math. Anal. Appl. 7 (1963), 284-288. DOI: 10.1016/0022-247X (63) $90053-2$.

[8] F. Mañosas and J. Villadelprat, Area-preserving normalizations for centers of planar Hamiltonian systems, J. Differential Equations 179(2) (2002), 625-646. DOI: 10.1006/jdeq.2001.4036.

[9] M. Sabatini, The period functions' higher order derivatives, $J$. Differential Equations 253(10) (2012), 2825-2845. DOI : 10.1016/j. jde.2012.07.013.

[10] M. Urabe, Potential forces which yield periodic motions of a fixed period, J. Math. Mech. 10 (1961), 569-578.

[11] M. Villarini, Smooth linearization of centres, Ann. Fac. Sci. Toulouse Math. (6) 9(3) (2000), 565-570. 
[12] G. Zampieri, On the periodic oscillations of $\ddot{x}=g(x), J$. Differential Equations 78(1) (1989), 74-88. DOI: 10.1016/0022-0396(89) 90076-4.

Dipartimento di Matematica Università di Trento

I-38123 Povo, (TN)

Italy

E-mail address: marco.sabatini@unitn.it 